\title{
Evaluation of Three Monte Carlo Estimation Schemes for Flux at a Point
}

\author{
H. J. Kalli* \\ E. D. Cashwell
}

"Visiting Staff Member. Helsinki University of Technology, Finland.

scientific Iaboratory

of the University of California

lOS ALAMOS. NEW MEXICO 87545

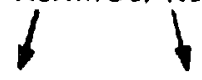

An Allitmalive Action fqual Opporlunily Employer 
Printed in the United States of America. Available from National Teshnical Informetion Service U.S. Department of Cominerece S28S Port Royal Roud Springficld, VA 22161

Pritc: Printed Copy gevo Microfiche \$3.úo 4.7

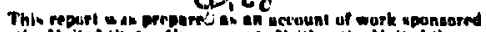

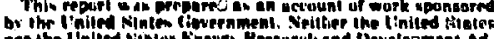

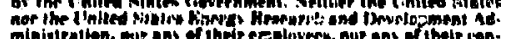

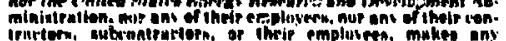

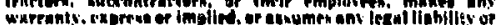

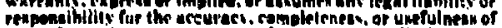

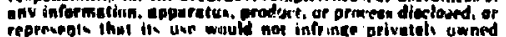

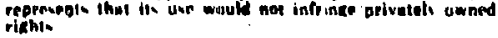




\title{
EVALUATION OF THREE MONTE CARLO ESTIMATION SCHEMES FOR FLUX AT A POINT
}

by

\author{
H. J. Kalli \\ E. D. Cachwell
}

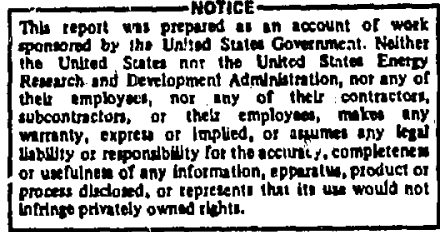

\section{ABSTRACT}

ABSTRACT

Three Monte Carlo estimation schemes were studied to avoid the difflculties caused by the $\left(1 / \mathbf{r}^{2}\right)$-singularity in the expression of the normal next-event estimator (NEE) for the fux at a point. We propose a new, fast, once-more collided flux estimator (OMCFE) scheme, based on a very simple probability density function (p.d.f.) of the distance to collision in the selection of the intermediate colligion points. This kind of p.d.f. of the collision distance is used in two nonanalng schemes using the NEE. In these two schemes, which have principal similarities to some schemes sroposed earlier in the literature, the $\left(1 / \mathrm{r}^{2}\right)$-singularity is canceled by incorporating the singularity into the p.d.f. of the collision points. This is achieved by playing a suitable nonanalog game in the neighborhood of the detector points.

The thrae schemes were tested in a monoenergetic, homogeneous infinitemodium problem, then were evaluaked in a point-cross-section problem us" ing the Monte Carlo code MCNG.
\end{abstract}

\section{INTRODUCTION}

The next-event estimator (NEE) used in a normal Monte Carlo game for the flux at a point has a severe limitation; namely, for a detector point in the scattering medium, the theoretical variance of the estimator is infinite even though the first moment is finite. This is due to the $\left(1 / r^{2}\right)$-singularity of the estimator: the NEE scores

$$
w \pi \frac{\operatorname{PSC}(\mu)}{2 \pi r^{2}} \exp \left\{-\int_{0}^{r} \Sigma_{s} d s\right\}
$$

for each collision point. Hese,

$r$ is the distance between the collikion point and the diatactor, 
$\mathrm{PSC}(\mu)$ is the probability density function (p.d.f.) for the cosine of the particle scattering angle; in the above expression $\mu$ is determined by the original direction before the collision and by the detector position,

WT is the particle weight (reduced by the probability of abrorption in the collision and multiplied by the number of neutrone coming out of the collision).

The exponential is the transmission probability from the collision point to the detector.

In practice, the singularity means that one collision close to the detector may have a very large contribution to the final result. As Kalog' has shown, the convergence of the next-event flux estimate toward the correct value is slower than the usual $1 / \sqrt{N}$ ( $N$ is the sample size):

The difficulty eaused by the $\left(1 / r^{2}\right)$-singularity in $\mathbf{E q}$. (1) can be avoided in several ways:

(1) If the detector points can be situated in a void outside of the scattering medium, the singularity no longer exists (because $r$ cannot $\rightarrow 0$ ). This is often the case, for example, in modeling many experiments, but in various other probleme the detectors simply cannot be moved out of the scattering medium.

(2) The $\left(1 / \mathrm{r}^{3}\right)$-singularity can be cut out by involving a suitable approximation for small values of $r$. For example, with the technique used in the Los Alamos code MCNG an imaginary sphere of radius $\rho$ is used around the detector point (cf. Corter and Cashwell ${ }^{2}$ ). Any collisions that occur outside this aphere are scored with $\mathrm{Eq}$. (1). For collisions within the sphere, the factor

$$
\left(1 / r^{2}\right) \exp \left\{-\int_{0}^{r} \varepsilon_{t} d s\right\}
$$

is replaced by its volume average, assuming uniform collisions within the sphere. The variance of this flux. estimator tends to decrease with increasing $\rho$; however, the approximation involved also becomes worse.

(3) Another approximate method is to use the track-length estimator in a small volume around the detector point. The estimation gives the average flux in this volume; hence, the approximation hecomes better as the volume becomes smaller. However, the smaller the volume, the less frequently it is hit by the particles, and the poorer the variance becomes.

(4) The singularity in the flux estimation can be avoided by using the adjoint calculation. This method is rather straightforward in a monoenergetic case, but is more complicated in energy-dependent problems. Further, if there are several point detectors, the calculation should be repeated for each detector. Finally, in the case of point sources this method leads to the same kind of singularity as in the direct calculation.

(5) The flux can be estimated without using the NEE. For example, in simple onecitmesisional geometries (infinite slab, sphere) the surface-crossing flux estimator can be used. In two-dimenaional $(x, y)$-geometry we can take (instead of a collision point) a colligion line parallel to the zaxis; the contribution from the collision to the detector point will be an integral from $-\infty$ to $+\infty$ ) along the line. This integral cannot be integrated in the general case, but it can be estimated by sampling a point on the line. By using a suitable p.d.f. for this point, one can derive an estimator for the flux that will have only a (1/b)-singularity, where $b$ is the distance from the datector point to the collision line. Hence, a new estimator with a less severe singularity is obtained.

The once-more collided flux estimators (OMCFE) by Kalos' also belong to this category. If the estimation of the once-more collided contribution is carried out for every collision point including the source, then the final estimate is for the collided flux. As we will show in Sec. III, the 
schemes involve the sampling of an imaginary intermediate collision point for every real collision. By choosing the p.d.f. of this intermediate point in suitable ways, estimators with only a $(1 / r)$-singularity can be derived. Consequently, the variance is finite and the convergence is $1 / \sqrt{\mathrm{N}}$.

During the course of this work Dubi and Horowitz published a papers in which they propose a new estimator called the track rotation estimator. It seems to avoid the $\left(1 / r^{2}\right)$-singularity, and the preliminary results are promising. However, the problems of implementing this technique in a general code are not known.

(6) We can use the NEE and cancel the singularity in Eq. (1) by incorporating the $\left(1 / r^{2}\right)$. singularity into the p.d.f. of collision points. Hence, a specially shosen nonanalog game is played in the neighborhood of the detector points. This idea was first propcsed by Steinberg and Kalos; Steinberg developed it further in later articles. ${ }^{.08}$ A different scheme of this type, suggested by Mikhailov," seems to have a (1/r)-singularity."*

Here we study three estimation schemes of the fifth and sixth categories above. We propose a new, once-more collided flux scheme which differs from those proposed by Kalos.' The scheme is based on a very simple p.d.f. of the path lengths in the sampling of the intermediatio collision points. In addition, we use this type of p.d.f. of the path length in two schemes of the sixth category basically similar to those proposed by Steinberg and Kalos' and by Steinberg. ${ }^{4}$ All these schemes will be studied first in a simple moncenergetic infinite-medium problem; und then they will be evaluated in a realistic point-cross-section problem using the Los Alamos Monte Carlo code MCNG. ${ }^{30}$

\section{RESELECTION}

Before studying the estimation schemes in detail, we will repeat the basic ideas of the special regeleciion technique proposed by Steinberg and Kalos ${ }^{4}$ for the local construction of the nonanalog p.d.f. for collision points.

We want to estimate with the Monte Carle ziteinod the integral

$$
\int_{V} G(x) f(x) d x,
$$

where $f(x)$ is a p.d.f. of $x$ in the dornain V. Let $V_{0}$ be a subdomain of $V$ and let $g(x)$ and $h(x)$ be two p.d.f.'s defined on the suhdomain $V_{0}$. Select the point $\mathbf{X}$ by the following procedure.

(1) Select a point $Y$ in $V$ using the original p.d.f. $f(x)$.

(2) If $Y$ is in $V_{0}$, reselect $X$ in $V_{0}$ using the p.d.f. $g(x)$.

(3) If $\mathbf{y}$ is not in $V_{0}$, let $X=Y$.

Then, an unbiased estimator $\mathrm{J}$ of

$$
\int_{v} G(x) f(x) d x
$$

is given by

-For Russian articles on the estimation of tlux at a point, see other volumes of $\mathrm{Zh}$. Vychisl. Mat. Mat. Fiz. 


$$
\begin{aligned}
& J=\frac{h(Y)}{f(Y)} \cdot \frac{f(x)}{g(X)} G(x), \text { if } x \text { is in } V_{0} \\
& J=G(x) \text {. if } x \text {. is not in } V_{0^{\prime}}
\end{aligned}
$$

Proof:

$$
\text { (J) }=\int_{V-V_{0}} G(x) f(x) d x+\int_{V_{0}} \frac{h(y)}{f(y)} f(y) d y \cdot \int_{V_{0}} \frac{f(x)}{g(x)} G(x) g(x) d x=\int_{V} G(x) f(x) d x \text {. }
$$

From the neutron transport point of view, $f(x)$ corresponds to the p.d.f. of a collision point in the phase space, and $G(x)$ to the "pay-off function" scored at the collision point, e.g., Eq. (1). In the following sections we will call the factor

$$
F=\frac{h(y)}{f(Y)} \cdot \frac{f(x)}{g(x)}
$$

the adjustment factor due to the raselection.

The p.d.f. $g(x)$ darines the sampling of points in $V_{0 ;}$ hence, the choice of $g(x)$ is restricted to some extent by t'ie local properties wunted for the p.d.f. in $V_{0}$. On the other hand, the choice of the p.d.f. $h(x)$ in $V_{o}$ is totally free. Siceinberg and Kalos' give some examples of the choices of $h(x)$; we vill repeat one of them here.

Examplos Let $h(x)=1 /\left|V_{0}\right|$, i.e., uniform distribution in $V_{o}$;

$$
\begin{aligned}
& \left|v_{0}\right|=\int_{v_{0}} d x \\
& F=T_{0}^{f}(x)
\end{aligned}
$$

\section{Application: A Directional Reselection Procedure}

Let us atudy the following procedure.

(1) Select the direction $\vec{\Omega}_{0}$ at a collision point $S$ by using the p.d.f. $f(x)=(1 / 2 \pi) \operatorname{PSC}(\mu)$. The direction $\vec{\Omega}_{0}$ is defined by the angles $\beta_{0}$ and $\phi_{0}$ (see Fig. 1 ).

(2) If $\vec{\jmath}_{0}$ is in the predefined cone, i.e., $\beta_{0}<\beta_{m}$, a new direction $\vec{\Omega}_{1}$ is reselected by sampling a new $\beta_{1}$ uniformly in $\left(0, \beta_{\mathrm{m}}\right)$ as well as a new polar angle $\phi_{2}$ uniformly in $(0,2 \pi)$.

(3) If $\vec{\Omega}_{0}$ is not in the cone, $\vec{\Omega}_{1}=\vec{\Omega}_{0}$, no reselection.

In this application the volume $V_{0}$ is the solid angle subtended by the cone. With the above procedure, the p.d.f. $g(X)$ is defined as

$$
g(x)=\frac{1}{2 \pi} \frac{1}{\beta_{m} \sin \beta_{1}} ; 0<\beta_{1}<\beta_{m} .
$$

By chocsing $h(Y)$ as in the preceding example we obtain

$$
h(Y)=1 /\left|v_{0}\right|
$$

where

$$
\left|V_{0}\right| \cdot \int_{\text {cone }} d \Omega \cdot 2 \pi\left(1-\cos \beta_{m}\right)
$$




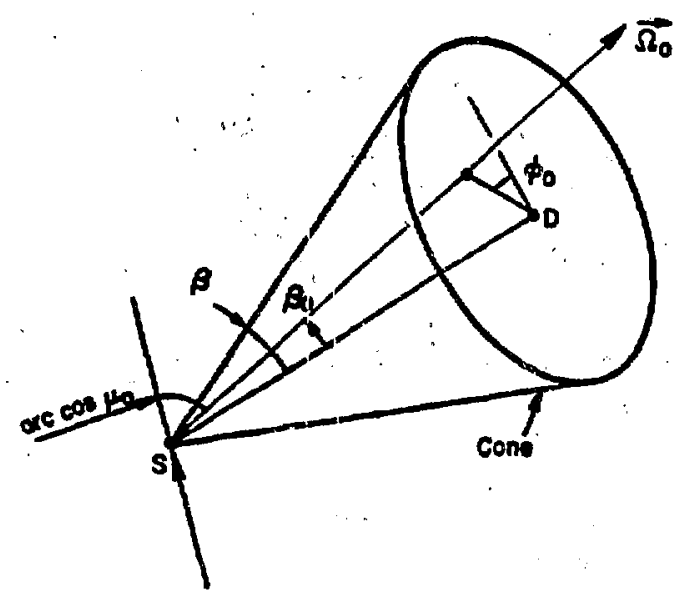

Fig. 1.

Geometry in the reselection of a direction.

Consequently, the adjustmen factor due to the directional reselection in the cone will be

$$
5_{\Omega}=\frac{h(y)}{f(\gamma)} \frac{f\left(x_{j}\right.}{g(x)}=\frac{B_{m} \sin \beta_{1}}{1-\cos \beta_{m}} \frac{\operatorname{PSC}\left(\mu_{1}\right)}{\operatorname{PSC}\left(\mu_{0}\right)} .
$$

where $\mu_{2}$ is the cosine cf the angle between the direction $\vec{\Omega}_{1}$ and the direction coming into collision at $\mathrm{S}$. The result, Eq. (3), which will be used later, is given by Steinberg.?

\section{ONCli-MORE COLLIDED FLUX ESTIMATOR (OMCFE)}

Kalog' auggests that the flux at a point be estimated by the "once-ricore collided" contribution. Starting from the expression of the once-more scattered flux contribution $\Phi_{1}$ due to a particle entering a collision, Kalos proposes two estimation schemes with only a $(1 / \mathrm{r})$-sirigularity. By starting from the expression of $\Phi_{1}$ due to one particle leaving a collision, another estimator of this type can be derived. We refer to Fig. 2; the particle leaves a collision at poist $S$, has an intermediate collision at $A$, and the detector is at $D$. TC get the expression for $\Phi_{4}$ we should integrate over all possible locations of the point $A$ on the half-line. In the simple case described in Ref. 1, with isotropic scattering and constant cross sections $\Sigma_{T}$ and $\Sigma_{\mathrm{B}}$, the expiession will be

$$
\phi_{1}-\int_{0}^{\infty} \varepsilon_{T} e^{-\Sigma_{T} s}\left(\frac{\Sigma_{S}}{\Sigma_{T}}\right) \frac{e^{-\Sigma_{T} r}}{4 \pi r^{2}} d s .
$$

Note that we have changed the meaning of $r$ slightly; it is now the distance between $A$ and $D$. Further, $B$ is the path length between $S$ and $A$, as can be seen in Fig. 2.

The normal way to estimate integral (4) would be to sample an intermediate point $A$, using the analog exponentiai p.d.f. of $\mathrm{s}$ :

$$
p(s)=\Sigma_{T} e^{-\Sigma_{T} s} .
$$




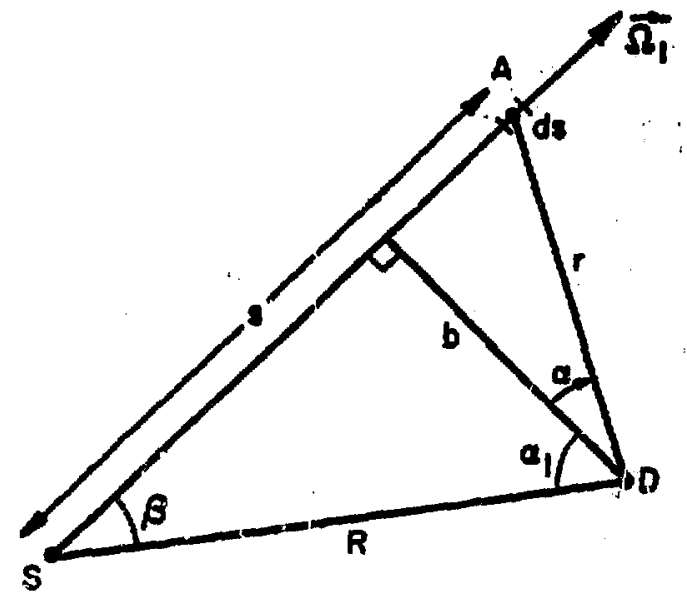

Fig. 2.

Geometry in the selection of the intermediate collision point $A$.

Then the estimator $\xi$ for $\Phi_{1}$ would be

$$
\varepsilon \cdot\left(\frac{\varepsilon_{S}}{\Sigma_{T}}\right) \frac{e^{-\Sigma_{T} r}}{4 \pi r^{2}}
$$

which of course is the normal NEE, Eq. (1), with the $\left(1 / r^{2}\right)$-singularity. However, instead of the analog p.d.f. p(s), we can use another p.d.f., say $\mathrm{p}^{*}(\mathrm{~s})$, and by choosing it carefully obtain a less severe singularity. The use of a nonanalog p.d.f. $\mathrm{p}^{*}(\mathrm{~g})$ is just 9 kind of importance sampling; hence, the new estimator, say $\xi^{*}$, for $\Phi_{1}$ would be

$$
\xi^{m}-\frac{p(s)}{p(s)} \xi=F_{s} \xi \text {. }
$$

We call the factor $F$, the adjustment factor due to the importance sampling of the path length 8 .

Example of $p^{*}(\alpha)$ : Select the intermediate point $A$ by sampling the angle $\alpha$ uniformly in $\left(\alpha_{1}, \pi / 2\right)$ (cf. Fig. 2). This scherne gives the following $\mathrm{p}^{*}(\mathrm{~s})$ :

$$
p(s)=\frac{b}{\left(\pi / 2-a_{1}\right) r^{2}} \cdot
$$

(Note that $\alpha_{1}$ is negative in Fig. 2.) The corresponding estimator $\xi^{*}$ is

$$
\xi^{*}=\frac{\left(\frac{\pi}{2}-\alpha_{1}\right) \Sigma_{S^{e}} \varepsilon^{-\Sigma_{T}(s+r)}}{4 \pi b} .
$$

(Another way to derive this estimator is to change the integration variable in Eq. (4) from $s$ to $\alpha$ and estimate the new integral by sampling $\alpha$. uniformly in the integration interval.) Instead of the $\left(1 / r^{2}\right)$-aingularity. we have now a less severe $(1 / b)$-singularity. In fact, for angles $\beta>\pi / 2$ it is 
relatively easy to see that the singularity is of the type (1/R) where $R$ is the distance between the points $S$ and $D(R$ is equal to our $r$ in Sec. I).

Further, for angles $\beta<\pi / 2$ we can use the directional reselection scheme introduced at the end of Sec. II. The cone is now a $2 \pi$ cone; $\beta_{m}=\pi / 2$. Each time we regelect the direction for sampling the point $A$, we should multiply the estimator $\xi^{*}$ of $\mathrm{Eq}$. (6) by the adjustment factor $\mathrm{F}_{\Omega}$ of Eq. (3). Hence, for $\beta<\pi / 2$ wo get.

$$
\xi^{*}=\frac{\pi}{2} \sin \beta=\frac{\left(\pi / 2-\alpha_{1}\right) \cdot \Sigma_{S} e^{-\Sigma_{T}(s+r)}}{4 \pi b}=\frac{\left(\pi / 2-\alpha_{1}\right) \cdot \Sigma_{S} \mathrm{e}^{-\Sigma_{T}(s+r)}}{8 R} .
$$

In sum, we propose a simple scheme for the selection of the intermediate collision point $A$ which leads to a (1/R)-singularity in the OMCFE. Note that the point $A$ is not a real collision point of the particle history; it is sampled just to estimate $\Phi_{L}$. Consequently, the sampling of $A$ causes no increase in the variation of the particle weight.

Note also that the intermediate collision point should be sampled separately for each detector; therefore, an increase in the computing time can be expected. However, far enough awey from the detectors the analog game can be used in selecting just one point $\mathrm{A}$. This $\mathrm{A}$ can also be used as the following real collision point. The scheme of Eqs. (6) and (7) is turned on just in a certain neighborheod of the detector points. We define this kind of modified scheme at the end of Sec. VI.

\section{ONOE-MORE COLLIDED FLUX ESTIMATOR (OMCFW) IN CODE MCNG}

In the general case we have a particle coming out of a collision at $\mathbf{S}$ in the direction $\mathbf{\Omega}$ witiu energy $E$. The once-more collided flux contribution $\Phi_{1}$ at the point $D$ and within the energy bin $\left(E_{1}, E_{s_{1}}\right)$ has now the following expression

$$
\begin{aligned}
& \Phi_{1}-\int_{E_{1}}^{E_{1+1}} \int_{0}^{\infty} \varepsilon_{T}(A ; E) P(S+A ; E) \times \\
& {\left[\frac{\Sigma_{e l}\left(A_{i} E\right)}{\Sigma_{T}\left(A_{i} E\right)} P S C_{e l}\left(\mu_{D}\right) q_{e\}}\left(E-E E^{\prime}\right)+\frac{\Sigma_{1 n}\left(A_{;} E\right)}{\Sigma_{T}\left(A_{i} E\right)} P S C_{i n}\left(\mu_{D}\right) q_{i n}\left(E+E^{\prime}\right)+\ldots\right.} \\
& + \text { (other collision types) }] \times \frac{M\left(A \rightarrow D_{j} E^{\prime}\right)}{2 \pi r^{2}} d s^{\prime} d E^{\prime} \text {. }
\end{aligned}
$$

As in Eq. (4), the integration in s goes over all the possible locations of the intermediate collision point A. (Note that almost all the functions and variables in Eq: (8) depend on the path length s.)

The notation for Eq. (8) (cf. Fig. 2) follows:

$\Sigma_{T}(A ; E)$ is the macroscopic total cross atetion at $A$ and at energy $E$,

$\Sigma_{\text {el }}(A ; E)$ is the macroscopic cross section of the elastic scattering at $A$ and $E$, $\Sigma_{1 n}(A ; E)$ is the macroscopic cross section of the inelastic scattering at $A$ and $E$, $P(S \rightarrow A ; E)$ is the transmission probability from $S$ to $A$ at energy $E$, $\mu_{D}$ is the cogine of the angle between the direction $\bar{\Omega}$ and the direction from $\AA$ to $D$, $\operatorname{PSC}_{\text {el }}(\mu)$ is the p.d.f. for the cosine of the elastic scattering angle, $\mathrm{PSC}_{\mathrm{In}}(\mu)$ is the p.d.f. for the cosine of the inelastic scattering angle, 
$q_{01}\left(E-E^{\prime}\right)$ is the s.d.f. for the energy $E^{\prime}$ in an elastic scattering at the point $A$ in the angle $\varepsilon_{i}$ $\cos \mu_{D}$ (a $\delta$-function),

$\mathrm{q}_{1 \mathrm{n}}\left(\mathrm{E} \rightarrow \mathrm{E}^{\prime}\right)$ is the same for the inelastic scattering:

$r$ is the distance between $A$ and $D$, and

$B$ is the path length from $S$ to $A$.

To describe how the right side of Eq. (8) car be estimated by using the scheme of Sec. III, we assume that a collision point $\mathbf{S}$ has just been sampled (i.e., the particle cosrdinates have been updated, the old direction and energy have been saved, the fraction captured has been subtracted from the weight, the collision type has been sampled as well as the new direction $\boldsymbol{\Omega}_{0}$ and the new energy $\mathrm{E}_{0}$ ). The estimation of the once-more collided flux at the point detectors is performed in the following steps:

(1) Save the collisicn parameters necessai $y$ to reconstruct the collision at $S$.

(2) Multiply the weight by the number of neutrons emerging from the collision.

(3). Select the next detector point.

(4) Calculate the angle $\beta_{0}$ (cf. Fig. 1).

(5) If $\beta_{0} \geq \pi / 2$, take $\vec{\Omega}_{1}=\vec{\Omega}_{0}, E_{1}=E_{0}$ ard go to step 9.

(6) If $\beta_{0}<\pi / 2$, reselect the direction $\vec{\Omega}_{1}$ (as described at the end of Sec. II).

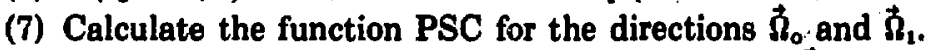

(8) Sample/calculate the energy $E_{1}$ in the direction $\widehat{\Omega}_{1}$.

(9) Sample the intermediate collision point $A$ on the half-line in the direction $\mathbb{Z}_{1}$ (Sec. III).

(10) Calculate the transmission probability from the point $S$ to the point $A$.

(11) Take $A$ as a collision point: sample the nuclide, subtract the fraction captured from the weight, and sample the collision type.

(12) Multiply the weight by the number of neutrons emerging from the collision at $A$.

(13). Determine the direction and the distance from the point $\mathbf{A}$ to the detector.

(1:) Calculate the function $\operatorname{PSC}\left(\mu_{D}\right)$ for the angle between the direction $\vec{\lambda}_{1}$ and the detector direction from $A$.

(15) Sample/calculate the corresponding energy $L_{L}$.

(16) Calculate the transmission probability from the point $A$ to the detector.

(17) Calculate the contribution (to the energy bin determined by $E_{D}$ ) using Eqs. (9) and (10) which are given below.

(18) If there are more detectors, go back to step (3).

(19) If there are no more detectors, reset the perticle parameters to correspond to the collision at the point $\mathbf{S}$.

(20) Continue the particle transport.

\section{Expressions for the Contribution}

By starting from Eq. (8) and by taking into account the scheme described as well as the adjustment factors due to the reselection of the direction and due to the importance sampling of the path length, we can derive the following expressions:

$$
\begin{aligned}
& B_{0} \geq \pi / 2: \text { Contribution }=W T \times \frac{\left(\pi / 2-a_{1}\right)}{2 \pi b} \cdot P\left(S+A ; E_{1}\right) \cdot P S C\left(\mu_{D}\right) \cdot P\left(A+D ; E_{D}\right) \cdot \Sigma_{T}\left(A ; E_{1}\right), \\
& B_{0}<\pi / 2: \text { Contribution }=W T \times \frac{\left(\pi / 2-a_{1}\right)}{4 R} \cdot \frac{\operatorname{PSC}\left(\mu_{g}\right)}{\operatorname{PSC}\left(\mu_{0}\right)} \cdot P\left(S+A ; E_{1}\right) \cdot \operatorname{PSC}\left(\mu_{D}\right) \cdot P\left(A+D ; E_{D}\right) \cdot \Sigma_{T}\left(A ; E_{1}\right)
\end{aligned}
$$


The notation has already been defined, except for $E_{\mathrm{D}}$ which is the energy after the particle has scattered at point $A$ in the direction of the detector and WT which is the final particle weight obtained in stops (2), (11), and (12) above. The scheme of the 20 steps may seem complicated, but most of the ateps already exist in the MCNG code. In fact, the deck needed to change the code to use the OMCFE scheme in the test problem (Sec. VII) consists of about 80 statement cards.

\section{TWO HONANALOG GAMES WITH THE NEXT-EVENT ESTIMATOR (NEE)}

In Sec. I listed six ways to avoid the $\left(1 / \mathrm{r}^{3}\right)$-singularity of the NEE in Eq. (1). The sixth category comprises nonanalog games; the singularity is canceled by incorporating the $\left(1 / \mathbf{r}^{3}\right)$ singularity into the p.d.f. of the collision points.

In Sec. III we proposed a simple nonanalog p.d.f. $p^{*}$ (s) for the path length s (cf. Eq. (5)). After a slight modification, this p.d.f. will a'so be used here. We refer to Fig. 3; a particle leaves a collision at $S_{1.2,}$, the direction $\vec{\Omega}$ of the particle hits the sphere $V_{0}$ around the detector $D$. Construct the nonanalog p.d.f. $\hat{p}(\mathrm{~g})$ to comply with the following rules:

(1) Sample a path length $s$ using the analog p.d.f. $p(s)$.

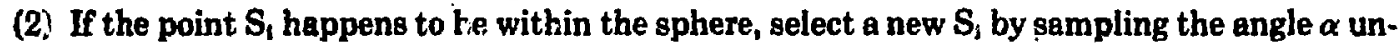
iformly in $\left(\alpha_{1}, \alpha_{s}\right)$.

Consequently, $p(s)$ is defineil as follows:

$\hat{p}(\mathbf{s})=\mathbf{p}(\mathbf{s})$ outside the sphere,

$=p_{1}(s)$ inside the sphere, where

$$
p_{1}(s)=\frac{p_{s}(t) b}{\left(a_{2}-a_{1}\right) r^{2}} .
$$

The angles $\alpha_{1}$ and $\alpha_{3}$ are denined in Fig. 3 (note that $\alpha_{1}<0$ in Fig. 3) and $P_{2}(\mathbb{R})$ is the analog probability of the point $S_{1}$ falling within the sphere (between the points $B$ and $C$ in Fig. 3). If the p.d.f. $\hat{p}(s)$ is used instead of $p(s)$ in the selection of the collision points in a Monte Carlo game, the particle weight should be adjr $\quad l$ for every collision in the sphere by the factor $p(\beta) / \hat{p}(s)=$ $p(s) / p_{1}(8)$, which compensates for thuc deformation in the true collision density. However, $p_{1}(s)$ in

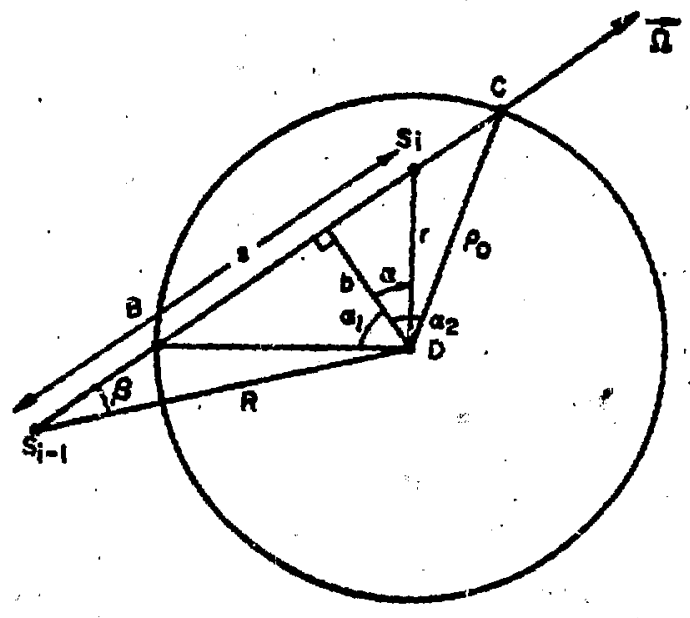

Fig. 3.

Geometry of the sampling schemes. 
Eq. (11) differs considerably from the analog exponential p.a.f. p(s); hence, the weight adjust. ment factor $p(s) / p_{2}(8)$ can cause a notable incresse in the variation of the weight.

All the properties of $p(s)$ are preserved and a smaller variation in the weight is obtained by using the following modified p.d.f.:

$\bar{p}(s)=p(s)$ outside the sphere,

= $p_{\mathbf{n}}(\mathbf{a})$ inside the sphere, where

$$
p_{2}(s)=Y p(s)+(1-\gamma) p_{1}(s) \text {. }
$$

Here, $\gamma$ is a paramster $(0<\gamma<1)$. The sampling from this new p.d.f. $\bar{p}(8)$, is very simple.

(1) Sampie a patk length s rising the analog p.d.f. $p(8)$.

(2) If the point $S_{1}$ happens to be within the sphere, accept the point with probability $\gamma$. With probability $(1-\gamma)$ use the uniform $\alpha$-sampling.

(The p.d.f. $\bar{p}(s)$ was proposed by one of the authors in Ref." 11 ; however, tha estimation scheme in that reference still has a (1/b)-singularity that was not found because of the notation used.)

We now define two nonanalog games that will have the desirer $\left(1 / r^{2}\right)$-singularity in the $p$,d.f. of collision points in a domain around the detector points. The first has similarities with the scheme in Ref. 4 , the second with the scheme in Ref. 7.

Schome 1. We assume first that the collision point $S_{101}$ already sampled is outside (or on) the sphere $V_{0}$ uround the detector. To select the point $S_{1}$, follow these steps:

(1) Sample $S_{1}$ normally, i.e., using the anulog game.

(2) If the point $S_{1}$ is outeide the sphere, accept it.

(3) If the point $S_{1}$ is inside the sphere, go back to $S_{1-1}$, reselect a new direction $\vec{\Omega}$ as described in Sec. II (the cone is defined by the point $S_{1-1}$ and the sphere $V_{0}$ ). Select a new point $S_{1}$ within the sphere and along the direction $\overrightarrow{\mathbf{n}}$ using the p.d.f. $p_{2}(s) / \mathbf{P}_{\mathbf{B}}\left(\vec{\Omega}_{1}\right)$.

If the preceding collision point $S_{1-1}$ is within the original sphere of radius $p_{0}$, wo have several possibilities. For example, we may let the radius $\rho$ of the sphere vary during the history: $p=$ Inin $\left(\rho_{o}, r_{m ! n}\right)$ where $r_{m / n}$ is the minimum distance from the collision points $S_{1}, S_{k, \ldots}, S_{1-1}$ to the detector $D$. Hence, $S_{t-1}$ will always be out of or on the sphere, and the point $S_{1}$ can be selected according to the three steps. However, in the preliminary calculations described in Sec. VI we obtained slightly better results using the following step (3) (steps 1 and 2 remain as above).

(3) If the point $S_{1}$ is in the sphere and $\sigma_{0}<\pi / 2$, carry out the directional reselection procedure of Sec. II with $\beta_{m}=\pi / 2$ (i.e., the cone comprises a half-space). Select the point $S_{1}$ within the sphere by using the p.d.f. $p_{2}(s) / P_{0}\left(\hat{\Omega}_{1}\right)$ for the distance to collision. If $\beta_{0}>\pi / 2$, no directional reselection is performed but the distunce to coilision is chosen from the p.d.f. $p_{2}(8) / \Psi_{4}\left(\hbar_{1}\right)$.

To compensate for the deformation in the true collision density, the particle weight should be adjusted by a factor for every collision within the sphere. If both $S_{1-1}$ and $S_{1}$ are in the sphere and $\beta_{0}>\pi / 2$, the adjustment factor is simply $\mathrm{p}(\mathrm{\theta}) / \mathrm{p}_{\mathrm{z}}(\mathrm{s})$. In all cases of directional reselection the ad. justment factor is more complicated and will now be derived using the theory developed in Sec. II.

The point $Y$ (sampled using the analog game) will have two coordinates-the direction $\vec{\Omega}_{0}$ and the path length $s_{0}$. Hence, the analog p.d.f. $f(\mathbf{Y})$ of $Y=\left(\boldsymbol{\Omega}_{0, g_{0}}\right)$ will be

$$
f(\gamma)=\frac{1}{2 \pi} \cdot \operatorname{PSC}\left(\mu_{0}\right) \cdot p\left(s_{0}\right)
$$

The reselcoted point $X$ will have the coordinates $\left(\vec{\Omega}_{1,}, \delta_{1}\right)$ and the p.d.f. of $X$ is

$$
g(x)=\frac{1}{2 \pi} \cdot \frac{1}{3_{n} \sin 8_{1}} \cdot \frac{P_{2}\left(s_{1}\right)}{P_{s}\left(\vec{n}_{1}\right)} .
$$


Finally, let us choose

$$
h(Y)=\frac{1}{2 \pi} \cdot \frac{1}{1-\frac{\cos B_{m}}{B_{s}\left(s_{0}\right)}} \cdot \frac{p\left(s_{0}\right)}{p^{\prime}}
$$

The adjustment factor, Eq. (2), of the purticle weight for collisions inside the sphere has now the following expression:

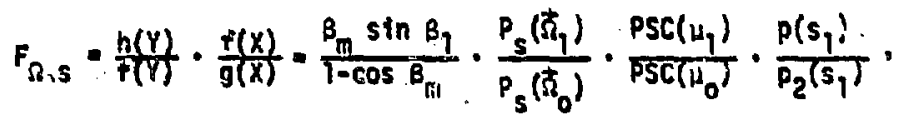

Close to a detector point the particlo weight will always contain a $\left(r^{2}\right)$-term which cancels out the dangerous $\left(1 / r^{2}\right)$-sirizularity in the NEE. The weak point of this scheme is in the factor $P_{0}\left(\vec{\Omega}_{1}\right) / P_{a}\left(\vec{Z}_{0}\right)$. We can see that a sphere is not a safe region $V_{o}$ arcund the detector, because the denominator $P_{0}\left(\vec{\Omega}_{0}\right)=C$ for $\vec{\Omega}_{0}$ a tangent of the sphere. In the calculations of Secs. VI and VII we, use an intersection of cone and sphere as given in Fig. 4. There are several ways to chonse $V_{0}$ and to avoid $P_{0}\left(\vec{\Omega}_{0}\right) \rightarrow 0$; some of them might give a faster execution of the scheme than orr choice does. We usc amaller sphere of fixed radius $p_{3}$ inside the original sphere of radius $\rho_{0}$ (as shown in Fig. 4) to avoid the difficulty $P_{1}\left(\vec{\Omega}_{0}\right) \rightarrow 0$. For example, in our calculations $\rho_{1} / \rho_{0}$ was chosen in the range 0.8 to 0.9 .

In the general case, the energy $\mathbf{E}_{0}$ in the direction $\lambda_{0}$ will differ from the energy $\mathbf{E}_{l}$ in the direction $\vec{\Omega}_{1} ;$ also, the total cross sections $\Sigma_{T}\left(E_{0}\right)$ and $\Sigma_{T}(E)$ might be totally different. Coissequently, the factor $P_{1}\left(\vec{n}_{2}\right) / P_{0}\left(\vec{n}_{0}\right)$ will vary considerably. sausing an increased variation of the particle weight.

The wealkness lurking in the factor $P_{0}\left(\vec{\Omega}_{1}\right) / P_{0}\left(\vec{\Omega}_{0}\right)$ can be avoided by using forced colligions within the volume $V_{0}$ but then an increase in the computing time can be expected.

The case of several point detectors does not require any special arrangements if the volumes $V_{0}$ are not overlapping. Overlapping volumes are discussed in Refs, 4 and 7 :

Scheme 2. 'I'he weakness of Scheme 1 also can be avoided by using a second scheme. Again, we assume first that the preceding collision point $S_{1-1}$ is out of the sphere $V_{0}$. Select the point $S_{1}$ in the following eteps.
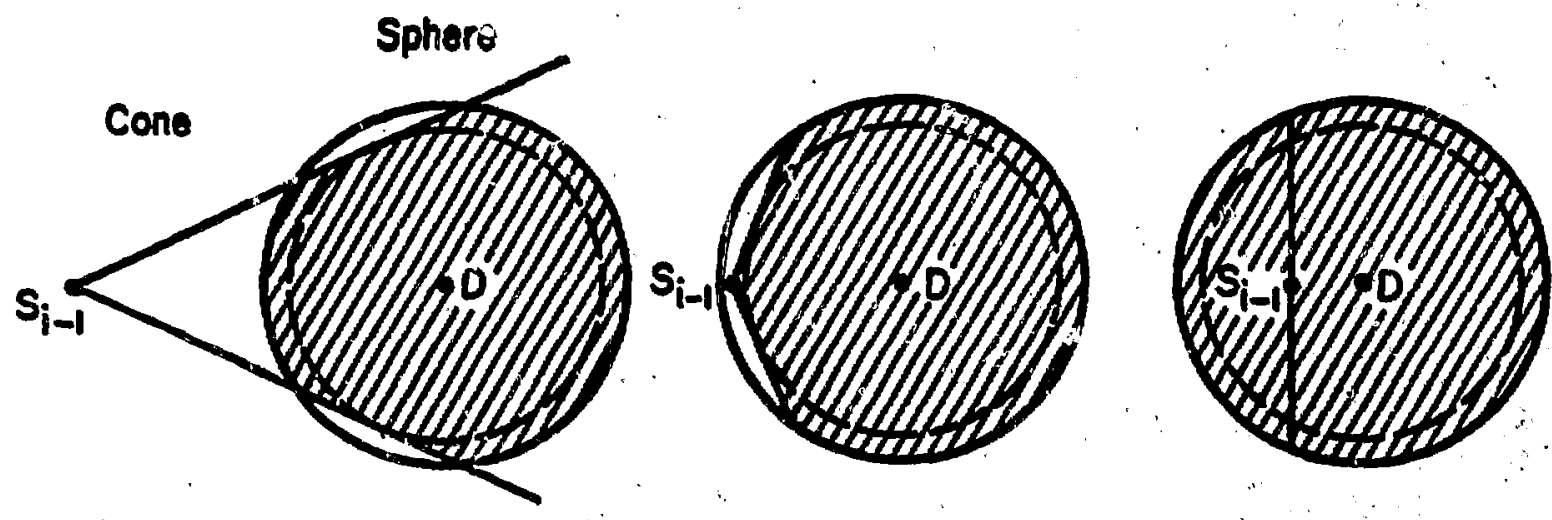

Nig. 4.

Reselection region. $V_{0}$ (shaded volume) in Scheme 1 for different locations of the collision point $S_{1-1}$. 
(1) If the direction $\vec{\Omega}_{0}$ after the collision at $S_{1-1}$ is in the cune defined by the point $S_{1-1}$ and tise sphere $V_{01}$ reselect the direction as described it the end of Sec. II.

(2) For the path lengtin s, use the normal p.d.f. $p(8)$ outgide the cone; inside the cone, sample $s$ from the p.d.f. $\overline{\mathbf{p}}(\mathbf{B})$.

Within the sphere, use the reselection of direction and the sampling from the p.d.f. $p_{2}(\theta) / P_{a}\left(\hat{\mathbf{n}}_{1}\right)$ whenever $\beta_{0}<\pi / 2$ (cE. Fig. 1); for $\beta_{0}>\pi / 2$ omit ths reselection of direction, as in Scheme 1 .

The particle weight should be adjusted by the factor $\mathbf{F}_{\Omega}$ of Eq. (3) every time the direction is reselected. To repeat,

$$
F_{\Omega}=\frac{B_{n} \sin B_{1}}{1-\cos \theta_{m}} \cdot \frac{\operatorname{PSC}\left(\mu_{1}\right)}{\operatorname{PSC}\left(\mu_{0}\right)} .
$$

Also, the weight should be multiplied by the factor $F_{a}=p(s) / p_{2}(8)$ every time the importance saunpling in the path-length selection is used. In total, we may have an adjustment factor of $F_{\Omega} \cdot F_{0}$ which is similar to $F_{\Omega}$ in Eq. (13) except that the dangerous factor $P_{0}\left(\vec{\Omega}_{1}\right) / P_{0}\left(\vec{\Omega}_{0}\right)$ is missing. Although the reselection is performed more frequently, a amaller variation in the particie weight might be expected.

In the case of several point detectors, special arrangements are needed because the direction $\overrightarrow{\boldsymbol{\Omega}}_{0}$ may be in the cone of several detectors simultoneously, or because the direction $\overrightarrow{\boldsymbol{R}}_{1}$ reselected with reference to one detector may lead to a colliaion close to another detector. If ihe volumes $V_{0}$ are not ovorlapping, one can always switch momentarily to Scheme 1. Steinberg ${ }^{7}$ has discussed the problem of several detectors. The treatment of several detectors will increase the variation of the particle weight.

We have described two nonaralog games, both of which avoid the $\left(1 / r^{2}\right)$-singularity in the NEE. This favorable property is obtained at the cost of increased variation in the particle weights; the increase (and the accuracy of the calculation) will depend on the number of detector points, their geometrical disposition in relation to each other and to the source region, the size of the spheres around detectors, and the free parameter $\gamma$ in $p_{2}(8)$.

\section{VL. PRELIMINARY CALCILATIONS}

To obtain preliminary information about the three estimation schemes, we made a series of calculations in a simple, monoenergetic, infinite-medium problem with isotropic scattering and with a point source at the origin. We esumated the collided flux at several distances from the origin, and compared the results with the exact values, which are calculated aising the results of nef. 12. In Schemes 1 and 2, the calculations were performed for several combinations of the free parameter $\gamma$ in $p_{2}(8)$ ind the radius $p_{0}$ of the imaginary sphere around the detector. To better reflect the real differences in the results, all the runs are correlated, in that the $n^{\text {th }}$ source particle of each problem operates on the same sequence of random numbers and these particles stay in phase unless significant changes occur in their histories.

Two values of the scettering probability $c$ were studied: $c=0.3$ and $c=0.9$. A typical set of regults for $c=0.9$ and for varying source-detector distance is concentrated in Figi. 6, 6, and 7 . These figures represent the estimated values for the collided flux and its relative error as a function of the sample size in the caiculation. In all the figures we hava first a standard run in which the normal NEF is used. This standard run utilizes analog sampling (except that obove the weight 0.2 capture is not a terminal event but is treated by reducing the weight and forcing the particle to scatter; below the weight of 0.2 capture is treated as a terminal event). The second curve is calculated by using histories identical to those for the curve NEE but using the OMCFE. 

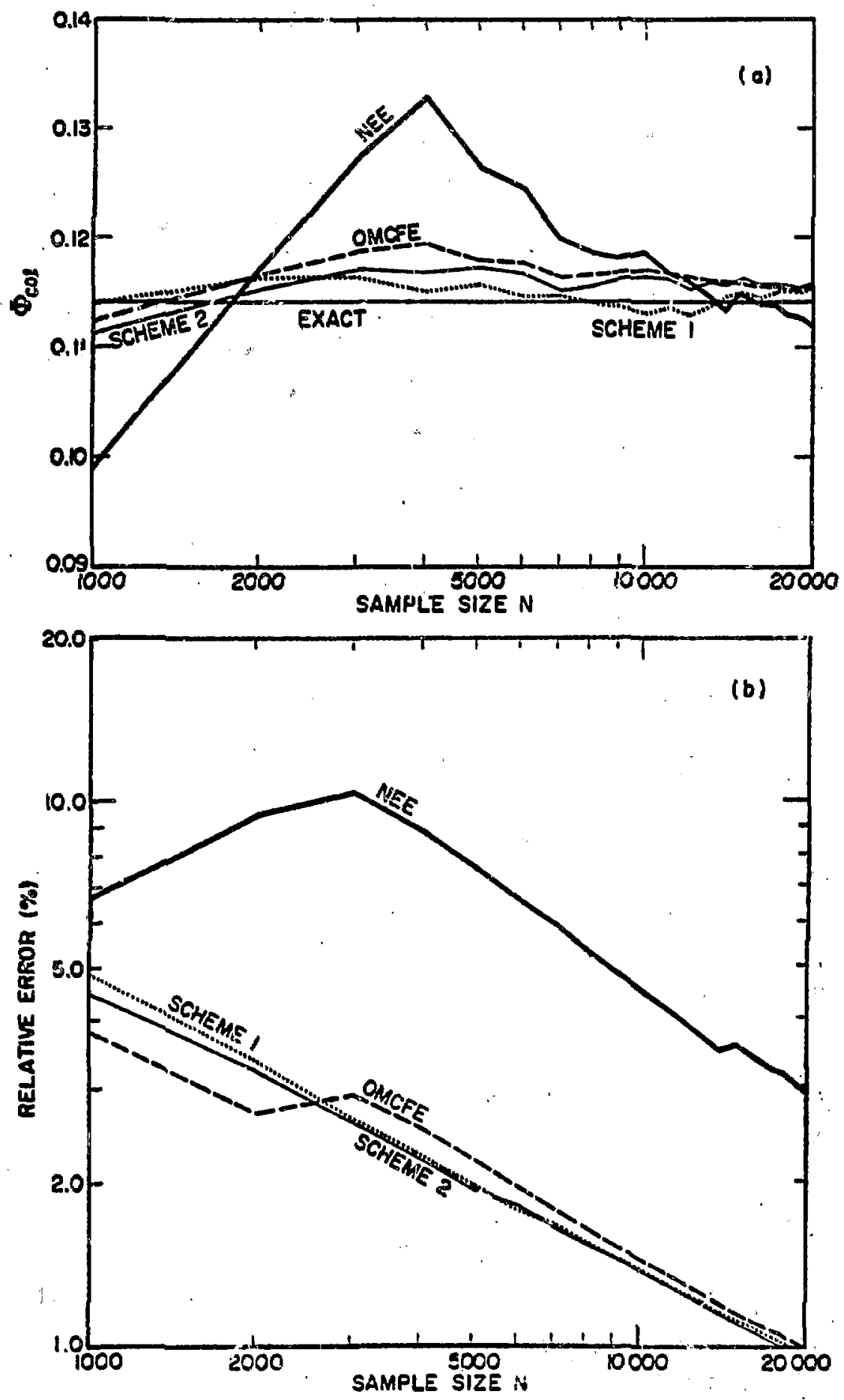

Fig. 5.

(a) Collided flux at a point $1 \mathrm{~m}$ fp from the source as a function of the sample size for different schemes, $c=0.9$. (b) Relative erroirs. 

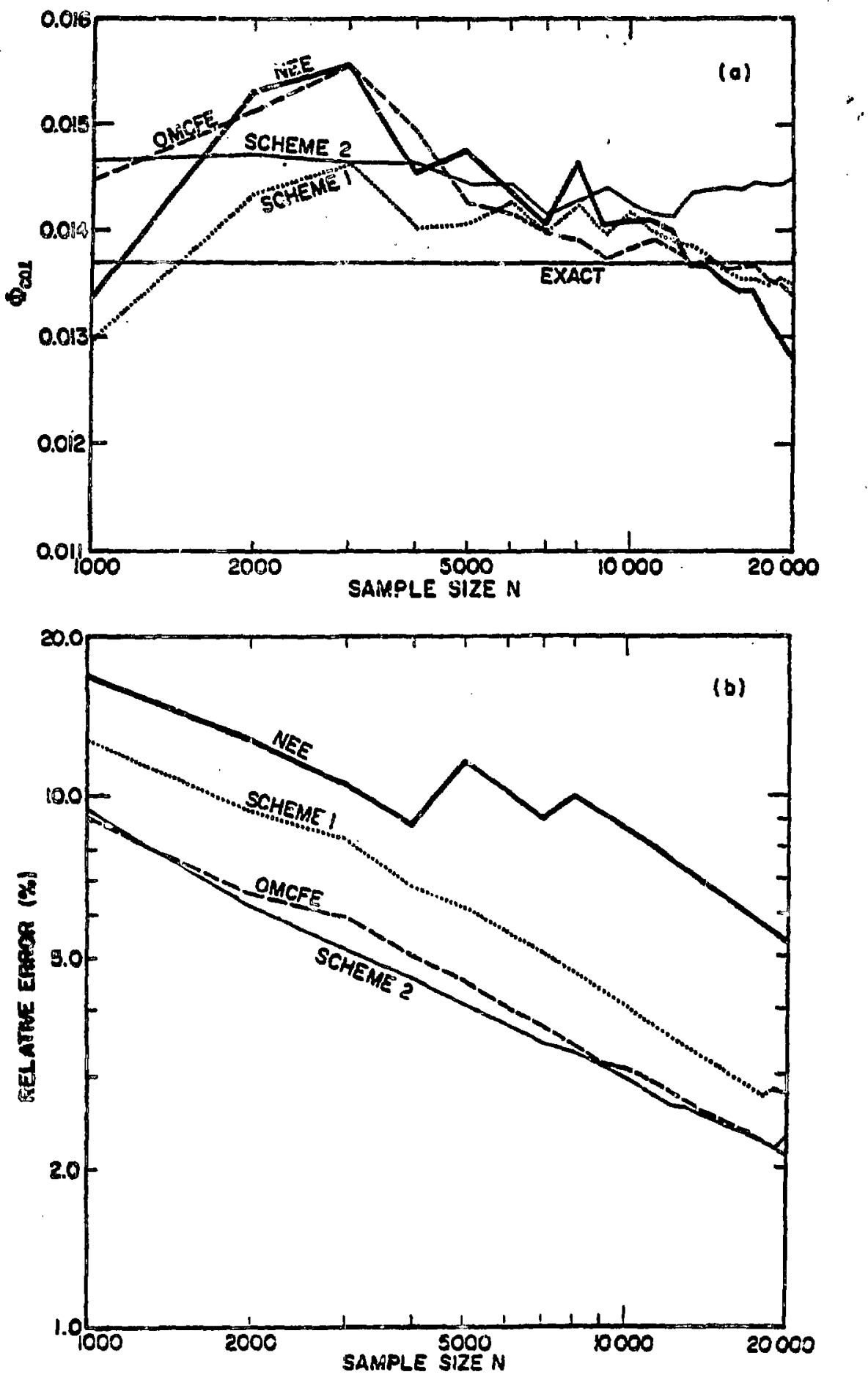

Fig. 6.

(a) Collided flux at a point $3 \mathrm{mfp}$ from the source as a function of the sample size for different schemes, $c=0.9$. (b) Relative errors. 

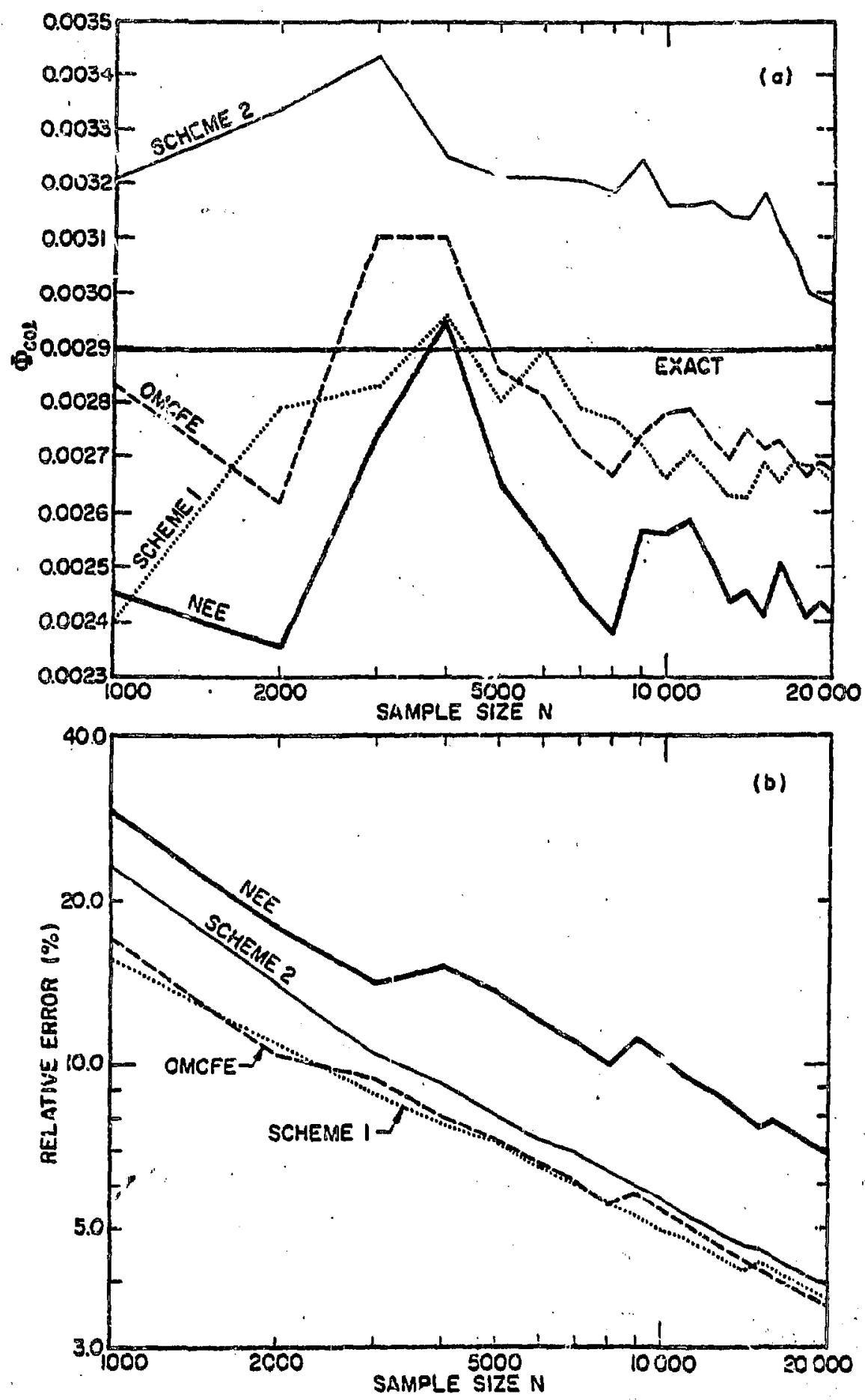

Fig. 7.

(a) Collided flux at a poin $5 \mathrm{mfp}$ from the source as a function of the sample size for different schemes, $c=0.9$. (b) Relative errors. 
In the two curves denoted by Scheme 1 and Scheme 2, the NEE is used together with the two nonanalog games of Sec. $V$.

Compazative results between the various methods of calculating flux at a pcint perhnps rhould not be tairon too literally, for, in a different problem or even in independent suns of the earse problem, slightly different comparisoss might bis obtained. However, the following trend seems evident. The estimated relative error is larger and its behevior is more irregular in the NEE case than in the other cases, reflecting the fact that the thecretical relative error is infinite in the NEE: case, whereas it is finite in the remaining three cases. On the other hand, the relative errors of the latter three schemes are more or less similar, and the behavior is something like $1 / \sqrt{N}$.

All the runs for Schemes 1 and 2 in Figs. 5, 6, and 7 have the sphers radius $p_{0}=2 \mathrm{mfp}$ and the fee parameter $\gamma=0.5$. The proper interval for the $\rho_{\mathrm{o}}$ was found to be 1.6 to $3.0 \mathrm{mfp}$, and for $\gamma$, 0.5 to 0.7 . Larger $\rho_{0}$ and/or smaller values of $\gamma$ increase the veriation of the particle weight; a small $\rho_{0}$ leads to results similar to those of the NEE.

The computational speed was 0 to $20 \%$ slower in Schemes 1 and 2 than in the NBE, depending on the position of the detector and on the radius $\rho_{0}$. The schenie using the OMCFE was $50 \%$ slower, as could be expected from the considerations of Sec. III. More speed can be gained by the following modification.

(1) Draw an imaginary sphere around the detector.

(2) If the collision point $S_{1}$ is in the sphere, calculate the once-more collided flux contribution as described in Sac. III.

(3) If the collision point $S_{1}$ is out of the sphere but the direction after the collision is within the cone defined by the sphere, calculate the once-more collided flux contribution by performisg the directional reselection in the cone.

(4) Otherwise, calculate the normal next-event contribution from the following collision point $\mathrm{S}_{1+1}$.

This modified scheme has the same accuracy as OMCFE for $\rho_{n}>2 \mathrm{mfp}$, but the computational speed is only 10 to $20 \%$ slower than in NEE.

\section{THE THREE SCHEMES IN THE CODE MCNG}

To study further the estimation schemes of Secs. III.V in a more realistic point-cross-section case, the following fictitious test problem was chosen (of. Figs. 8, 9, and 10). A source of 1-MeV neutrons sampled uniformly in volume is started in a 5 -cm-radius carbon sphere surrounded by a spherical, 20-cm-thick iron shell. The neutron flux within the energy bin 0.2 to $1.0 \mathrm{MeV}$ is es. timated at various points in the iron shell. The problem has spherical symmetry; hence, the "exact" values can be calculated with high accuracy by utilizing the eurface croseing flux entimator. Because of the large variation of the iron total cross section within the given energy bin, this problem is a severe test for the schemes.

Implementation of the schemes into the code MCNG for the solution of the test problem is relatively straightforward; as marisioned at the end of Sec. IV, several parts of the schemes already exist in the code and can be used just by changing the flow of the execution. Scheme 1 (Sec. V) needs more explanation: In this scheme we should go back to the preceding collision point $S_{1-1}$, if the point $S_{1}$ is found to be within the volume $V_{0}$. All the information required to reconstruct the collision at $S_{1+1}$ is saved in $M C N G$, except for information on a particle coming from the bank. Hence, a new array is needed to save this collision data for the banked particles.

A set of flux results at three different points in the iron shell is concentrated in Figs, 8, 9, and 10. The runs are basically identical, except for the differences in the schemes. The same sequence of random numbers is used, but the particles no longer stay in phase; therefore, the 

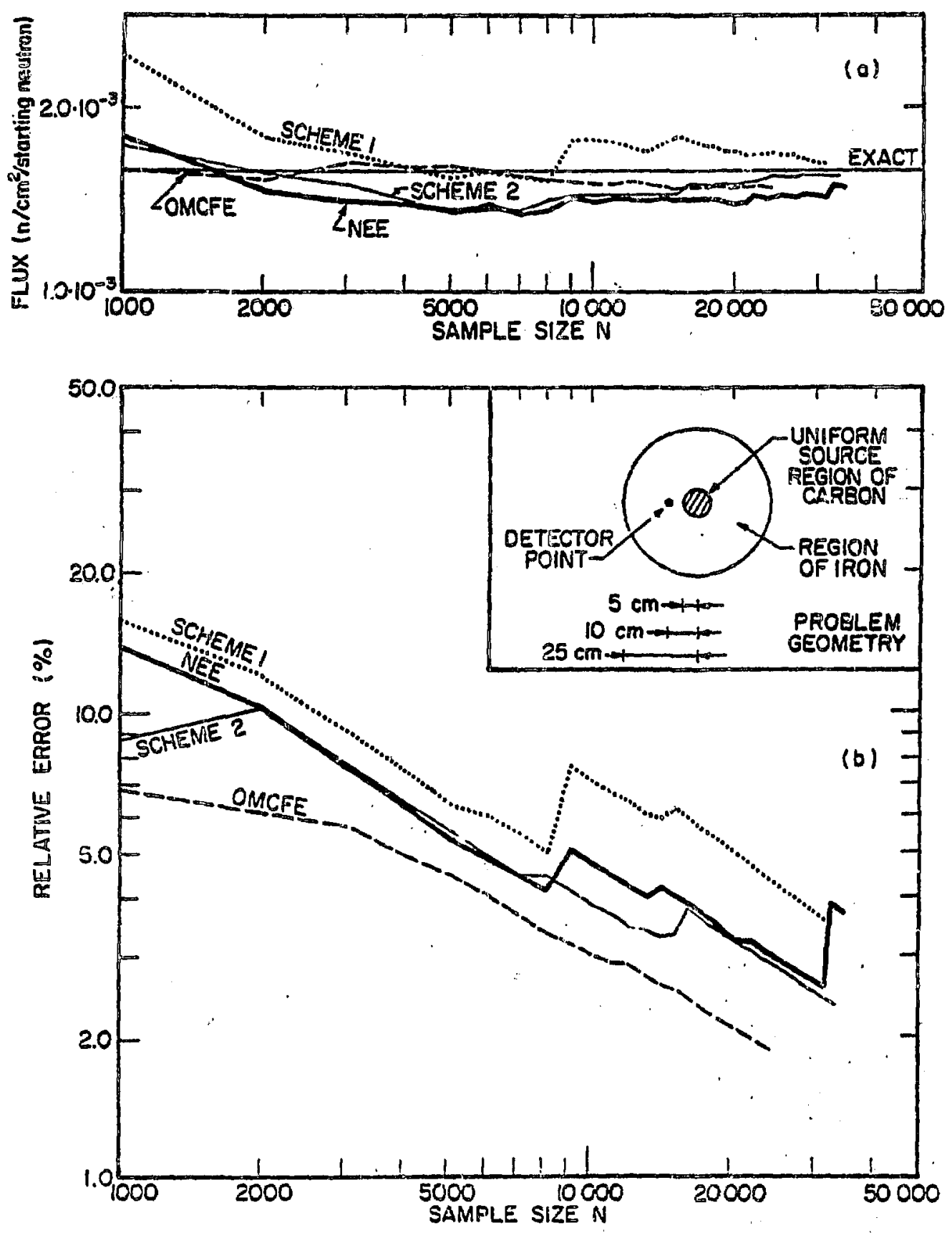

Fig. 8.

(a) Neutrai flux by different schemes at a paint $10 \mathrm{~cm}$ from the center of the system.

(b) Relative errors. 


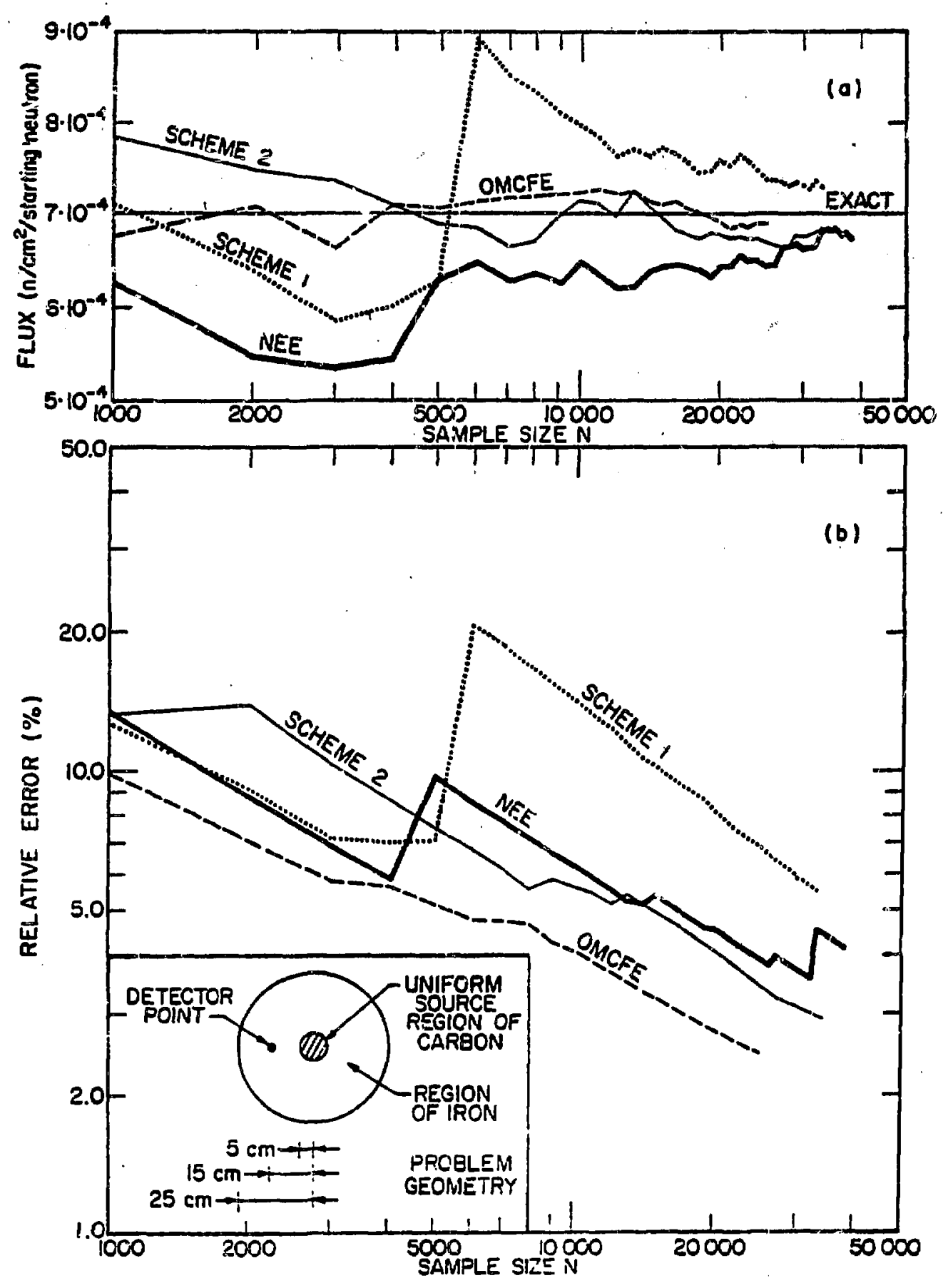

Fig. 9.

(a) Neutron flux by different schemes at a pnint $15 \mathrm{~cm}$ from the center of the system.

(b) Relative errors. 

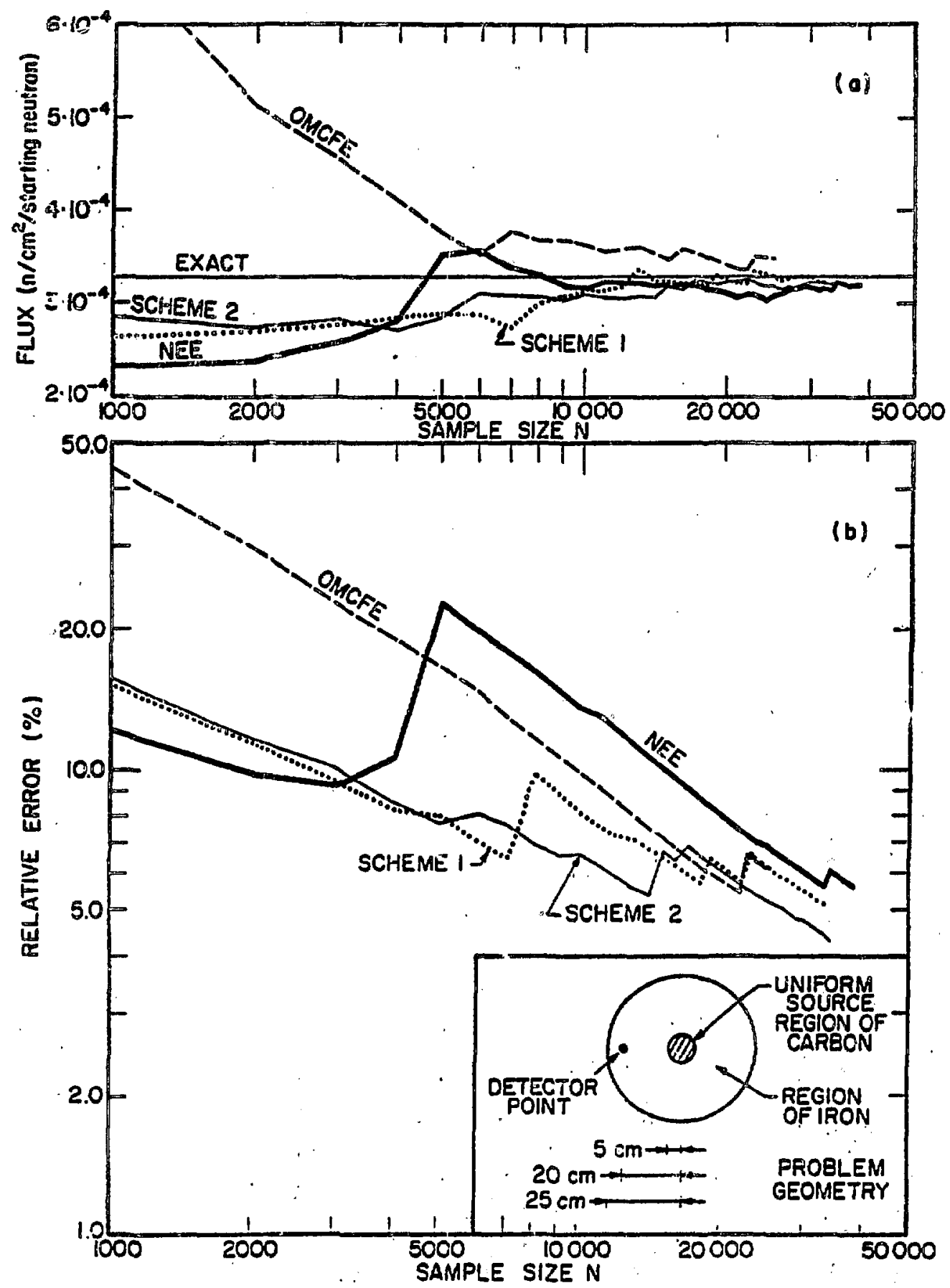

Fig. 10.

(a) Neutron flux by different schemes at a point $20 \mathrm{~cm}$ from the center of the system.

(b) Relative errors. 
correlation between the results is much weaker than in Sec. VI. The parameter $\gamma=0.5$ in all Scheme 1 an 12 runn, with the radius $\rho_{0}=3.0 \mathrm{cr}$ in Fig. $B$ and $5.0 \mathrm{~cm}$ in Figs. 9 and 10 .

Because of the nature of the comparative results, we wam against drawing hard and fast con. ciusions. However, this test problem seems to reveal the inner weakness of Scheme 1 discussed in Sec. $V$, but not seen in the results if the preliminary calculations in Sec. V. Because of the large and rapid variations in the iron total crose sfetion, the factor $P_{2}\left(\overrightarrow{\mathbf{R}}_{1}\right) / \mathbf{P}_{0}\left(\overrightarrow{\mathbf{R}}_{0}\right)$ in $\mathbf{E q} .(13)$ causes an increased variation of the partisle weight. Further, the relative error calculated from the NEE results tends again to have larger values, even if there are no jumpe in the NEE fluxes. In Fig. 10 the OMCFE results begin very poorly, although they later come close to the othor curves. This reminds us of the fact that the OMCFE atill has a ( $1 / \mathrm{r}$ )-singularity. (With another sequence of random numbers a nicer result was obtained.)

In all the cornputer runs of Figs. 8, 9, anc 19 we had one point detector at a time. The execution speed was slower by only $\sim 4 \%$ in Schemes 1 and 2 than in NEE; on the other hand, the OMCFE scheme of Sec. IV was $\sim 30 \%$ slower. However, with the modified scheme dencribed at the end of Sec. VI, a computing speed only $\sim 8 \%$ slower was abtained without any significant loss in accuracy.

\section{CONCLISSIONS}

We now list several key aspects of the three schemes for the estimation of the flux at a point.

Singularty. The OMCFE has a $(1 / r)$-singularity, whereas in Schemes 1 and 2 the $\left(1 / r^{2}\right)$ singularity of the NEE is cancelled by the factor $r^{2}$ in the particle weight.

Implementation. The implementation of the schemes into the code MCNG for the solution of the test problern described in Sec. VII was relatively straightforward in all three cases.

Accuracy. Although one must be careful in making dogmatic statements regarding the comparisons shown in Figs. 5-10, several points should be made. In these calculations, the OMCFE scheme seems no worse than Schemes 1 and 2 , in spite of the (1/r)-singularity. At a point close to a void boundary the OMCFE scheme might become less accurate because many intermediate collision points are lost in the void. This can be avoided by a slight change in the scheme. Scheme 1 is subject to large weight fluctuations in the case of rapidly varying total crose sections. This dunger is alleviated in Scheme 2. More important than a comparison of these three methods is the fact that in general they gave satiafactcry results, with errore of a few percent (ugually $\sim 2$ to $5 \%$ ) in the known answer, in $\sim 4$-min runs on the CDC-7600.

Computational Speed. After the modification of the OMCFE scheme described at the end of Sec. VI the differences in the computational speed are only a few percent.

Particle Weight Variation. Because the intermediate collision points in the OMCFE scheme are selected just for the calculation of the contribution and because this operation leaves the particle history unchanged, the $\mathrm{OMC} \mathrm{E}$ scheme causes no extra variation in the particle weight. On the contrary, in Schemes 1 and 2 the nonsingularity is obtained at the cost of the increased variation in the particle weight. This increase (and consequently the accuracy of the calculation) depends on the number of the detector points, on their geometrical disposition in relation to each other and to the source, as well as on the values of the free parameters $\gamma$ and $p_{0}$. The effect of rare high-weight particles can be moderated by splitting. 
The Case of Several Point Detectora. The estimation of the flux simultaneously at several points causes no problems in the OMCFE scheme. The same is true in Scheme 1 if the volumes $V_{0}$ are not overlapping. In Scheme 2 a special treatment is needed in the case of several detectors

Compatibility with the Osher Estimatore of the Code. The code MCNG has other optional estimators (such as the track-length estimator). which can be used simultaneously with the fluxat-a-point estimator. Implementation of the OMCFE scheme catises no troubles in this respect; on the other hand, the increased variatics of the particle weight in Schemes 1 and 2 tends to decrease the accuracy of the other estimators.

\section{REFERENCES}

1. M. H. Kalos, "On the Estimation of Flux at a Point by Monte Cario, "Nucl. Sci. Eng. 16, 111 117 (1963).

2. L. L. Carter and E. D. Cashwell, Particle-Transport Simulation with the Monte Carlo Method, ERDA Critical Review Series (1975).

3. A. Dubi and Y. S. Horowitz, "The Monte Carlo Track Rotation Estimator," 5th Int. Conf. Reactor Shielding, Knoxville, TN, April 18-23, 1977.

4. H. A. Steinberg and M. H. Kalos, "Bounded Estimators for Flux at a Point in Monte Carlo," Nucl. Sci. Eng. 44, 406-412 (1971).

5. H. Steinberg and H. Lichtenstein, "Implementation of Bounded Point Estimators in Foint Cross-Section Monte Carlo Codes," Trans. Am. Nuc\}. Soc. 17, 259-260 (November 11-15, 1973).

6. H. Steinberg, "Implementation of Improved Bounded Estimation of Flux-at-a-Point for Several Detectors," Trans. Am. Nucl. Soc. 19, $4 \div 2$ (1974).

7. H. A. Steinberg, "Bounded Estimation of Flux-at-a-Point for One o: More Detectors," Proc. NEACRP Mceting of a Monte Carlo Study Group, Argonne National Laboratory, july 1-3, 1974.

8. H. Steinberg, "Bounded Estimation of Flux-at-a-Point Near Region Boundaries," Trans. Am. Nucl. Soc. 23, 607-608 (June 14-18, 1976).

9. G. A. Mikhailov, "Modificaition of the Local Estimate of Particle Flow by Monte Carlo Method," Zh. Vychisl. Mat. Mat. Fiz. 13, 3, 574-582 (1973); Engl. trans. in U.S.S.R. Comput. Math. Mat. Phys. 13, 3, 45-55 (1973).

10. L. L. Carter, E. D. Cashwell, C. J. Everett, C. A. Forest, R. G. Schrandt, W, M. Taylor, W. L. Thompson, and G. D. Turner, "Monte Carlo Code Development in Los Alamos, Los Alamos Scientific Laboratory report LA-5903-MS (March 1975). 
11. H. Kalli, "On the Variance Reduction Techniques in Monte Carlo Solutions of Neutron Transport Problems," Acta Polytech. Scand. Ph 89 (1972), 27 pp.

12. K. M. Case, F. DeHoffman, and G. Placzek, Introduction to the Theory of Neutron Diffusion, Vol. I (Los Alamos Scientific Laboratory, 1953). 